\title{
Subcutaneous Panniculitic T-Cell Lymphoma in an Adolescent: A Case Report
}

\author{
Paula Bianca E Nuquiłand Flerida G Hernandez
}

University of Santo Tomas Hospital, Espana, Manila, Phillippines

\author{
${ }^{*}$ Corresponding author \\ Paula Bianca E. Nuqui, University of Santo Tomas Hospital, Espana, Manila, \\ Phllippines
}

Submitted: 20 Dec 2019; Accepted: 27 Dec 2019; Published: 26 Feb 2020

\begin{abstract}
Rationale and Objectives: Subcutaneous panniculitic T-cell lymphoma (SPTCL) is a rare neoplasm accounting for less than 1\% of pediatric Non-Hodgkin Lymphomas. It is most common in young adults with predominance of female cases at 0.5. Symptoms include multiple nodules involving the subcutaneous tissues of the extremities and trunk, neck and face. B symptoms such as fever, chills, night sweats and weight loss, have been reported. Rare extra-cutaneous manifestations include edema, involvement of the bone marrow, lymph nodes, liver, spleen, lungs and viscera. Laboratory abnormalities such as cytopenias and elevated lactate dehydrogenase have been recorded. Hemophagocytic syndrome (HPS) occurs in 33\% of cases, which were correlated with fatal outcome.
\end{abstract}

Case Report: We present a rare case of a 17 year-old male diagnosed with subcutaneous pannicultic T-cell lymphoma manifesting with prolonged fever, weight loss, and multiple subcutaneous nodules. He also presented with extra-cutaneous manifestations of facial edema, pleural effusion and ascites, lymph node enlargement, hepatosplenomegaly with jaundice and pancytopenia.

Conclusion and Summary: Subcutaneous Panniculitic T-Cell Lymphoma may present with an unusual finding of facial edema, ascites and pleural effusion, lymph node enlargement, hepatosplenomegaly with jaundice and pancytopenia alongside skin manifestations of subcutaneous panniculitis. HPS may complicate the course, treatment decisions and outcome of the disease.

\section{Introduction}

Subcutaneous panniculitic T-cell lymphoma (SPTCL) is a rare neoplasm accounting for less than $1 \%$ of pediatric Non-Hodgkin Lymphomas [1].

It is most common in young adults with a median age of 36 years (range $9-79$ years), 19\% of whom are patients younger than 20 [2]. There is female predominance with a male to female ratio of 0.5 [2]. The usual clinical manifestations include multiple nodules involving the subcutaneous tissues of the extremities and trunk, but can also involve the face and neck. B symptoms such as fever, chills, night sweats and weight loss have been reported in two-thirds of cases [2]. Edema is rarely seen with only three reported cases presenting with facial edema at onset. Rare extra-cutaneous manifestations involve the bone marrow, lymph nodes, liver, spleen, lungs and viscera $[2,3]$.

The presence of cytopenias and elevated lactate dehydrogenase have been recorded [3]. Hemophagocytic syndrome (HPS) accompanying SPTCL occurred in $33 \%$ of cases, which was correlated with poor outcome [4].

We report a rare case of subcutaneous pannicultic T-cell lymphoma occurring in a 17-year-old male presenting with multiple subcutaneous nodules on extremities, trunk and face associated with facial edema, pleural effusion and ascites, lymph node enlargement, hepatosplenomegaly with jaundice and pancytopenia.

\section{Case Summary}

A 17-year-old male presented with multiple painless hyperpigmented nodules on his right thigh measuring $0.5 \mathrm{~cm}$ accompanied by bilateral peri-orbital edema of two months. It was preceded a week before by fever, decreased appetite, weight loss, joint pains, and night sweats. He was initially treated by his primary physician for renal cause of symptoms without improvement. On physical examination, he had multiple erythematous, some hyperpigmented or violaceous, subcutaneous nodules on his extremities, trunk and face. His face was edematous and scaly on both mandibular areas (figure A). He had multiple lymphadenopathies on the cervical, submandibular, right supraclavicular area, axillary, epitrochlear and inguinal areas measuring 1 to $2 \mathrm{~cm}$ in diameter, hepatomegaly and splenomegaly. Hemoglobin was $108 \mathrm{gm} / \mathrm{L}$, hematocrit $31 \mathrm{vol} \%$, WBC $3.5 \times 10^{9} / \mathrm{L}$, platelet count $234 \times 10^{9} / \mathrm{L}$, albumin level $2.59 \mathrm{~g} / \mathrm{L}$, total bilirubin $6.09 \mathrm{mg} / \mathrm{dL}$, direct bilirubin $5.68 \mathrm{mg} / \mathrm{dL}$, creatinine $0.56 \mathrm{mg} / \mathrm{dL}$ and SGPT 149.1 IU. Computed tomography scan of the chest and abdomen showed subcutaneous soft tissue edema involving multiple cervical neck spaces, facial and clavicular regions, minimal pleural effusion, hepatosplenomegaly, ascites, and subcutaneous soft tissue edema at the lower lumbar areas. CSF analysis was negative. Bone marrow core biopsy demonstrated variable cellularity of $10-50 \%$; lymphoid aggregates were not seen. The subcutaneous 
nodule histopathologic results revealed atypical lymphocytes with adipotropism. Immunohistochemical stain revealed CD3+, CD20and $\mathrm{Ki} 67$ at 70-80\% (figure $\mathrm{C}$ and D), which were consistent with the diagnosis of subcutaneous panniculitic T-cell Lymphoma.

He was initially given Prednisone $60 \mathrm{mg} / \mathrm{m}^{2}$ and was lost to follow up due to significant clinical improvement. He came back a month later and was given doxorubicin. Vincristine was withheld due to persistent direct hyperbilirubinemia with noticeable icterisia and jaundice. He was aggressively managed twice for febrile pancytopenia after the first and once after the fourth doxorubicin dose. There was significant resolution of previously visible and palpable subcutaneous panniculitits and edema, however, hepatosplenomegaly remained. (Figure B).

Hemophagocytic syndrome was entertained because of persistent bicytopenia with hemoglobin 82.5 gms/L, hematocrit $25 \mathrm{vol} \%$, WBC $5 \times 10^{9} / \mathrm{L}$ and platelet $40 \times 10^{9} / \mathrm{L}$, recurrent febrile episodes and persistent hepatosplenomegaly with jaundice despite doxorubicinprednisone treatment. Works-ups showed elevated serum ferritin at $61,124 \mathrm{ng} / \mathrm{L}$ and low fibrinogen at $0.23 \mathrm{~g} / \mathrm{L}$, however, triglyceride was normal at $33.6 \mathrm{mg} / \mathrm{dL}$. Whole abdominal ultrasound revealed hepatosplenomegaly with diffuse fatty infiltration of the liver. Liver biopsy revealed tissue strips with moderate steatosis and no evidence of lymphoma.

The blood count eventually improved and he was placed on intensification phase (based on ALL regimen) with cyclophosphamide, 4-day weekly cytarabine subcutaneous injection and daily oral mercaptopurine. On the third week of cytarabine, he developed febrile pancytopenia accompanied by hemoptysis and melena. Despite aggressive treatment with broad-spectrum antibiotics, blood component therapy and GCSF, he developed severe pneumonia and respiratory failure leading to death.

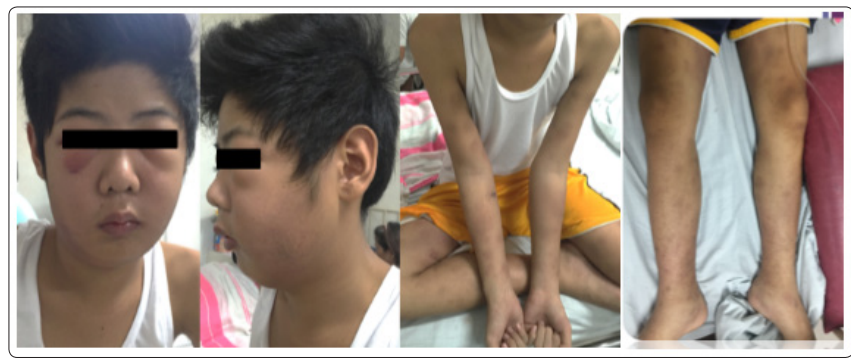

Figure A: Clinical picture before induction chemotherapy

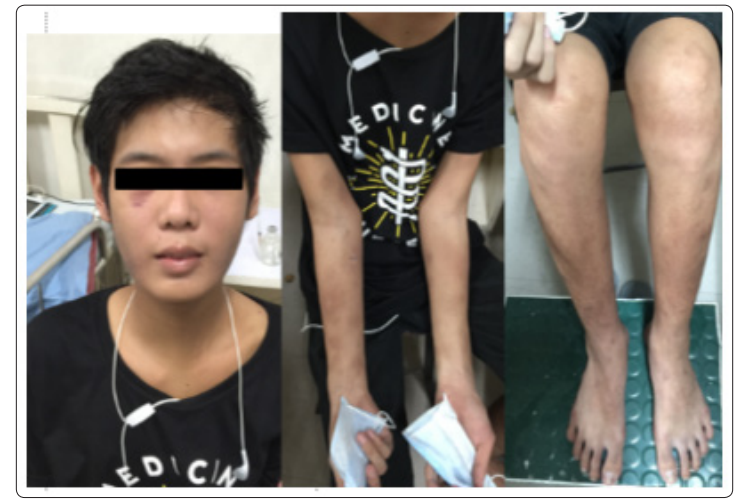

Figure B: Clinical picture after induction chemotherapy

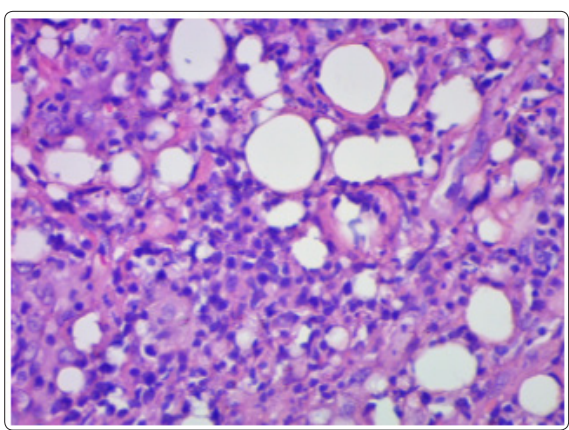

Figure C: H \& E stain of subcutaneous nodule showing atypical lymphoid infiltrates surrounding adipocytes

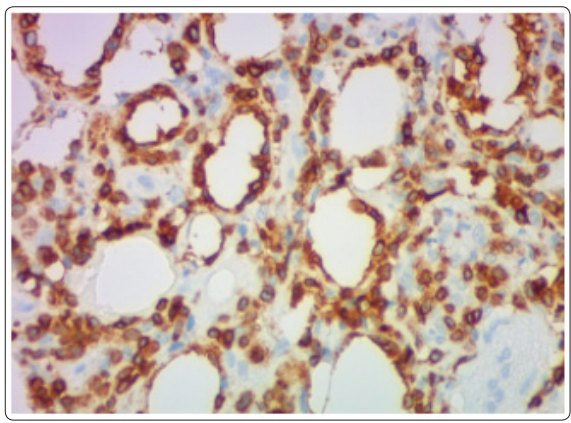

Figure D: CD3 staining of subcutaneous nodule showing atypical lymphocytes with adipotropism

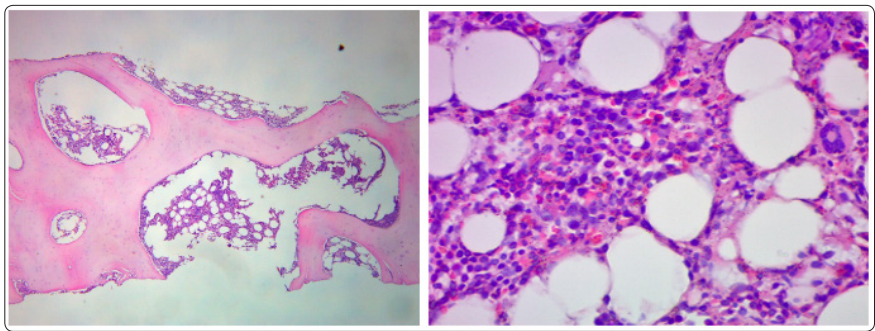

Figure E/F: Bone marrow core biopsy showed variable cellularity of $10-50 \%$ with no lymphoid aggregates

\section{Discussion}

Subcutaneous panniculitic T-cell lymphoma (SPTCL) is defined as a cytotoxic T-cell lymphoma described as subcutaneous infiltration of small to large pleomorphic T-cells and macrophages, which can predominantly affect the legs. SPTCL usually affects people after the second decade of life. Of the 21 cases seen at the Mayo clinic from July 1973 to June 2004, the median age of diagnosis was 42 years (range 23-80 years) and 15 (71\%) were women [3].

From the European Organization, Research and Treatment of Cancer, Cutaneous Lymphoma Group Study published in the Blood Journal, 2008 , only $19 \%$ of 83 cases of SPTCL was 20 years or younger [2].

O'suoji et al (2016 p.795) from the Children's Oncology Group (COG) Study on Rare Pediatric Non Hodgkin Lymphomas (NHL) participated by $100 \mathrm{COG}$ institutions reported only a total of 11 diagnosed cases of SPTCL from a total of 142 who were diagnosed with primary cutaneous NHL from May 2005 to December 2013. The reported cases were mostly female $(73 \%)$ and none of them had marrow, brain or lymph node involvement [5]. 
Patients with SPTCL generally present with solitary or multiple nodules and plaques, which mainly involve the legs, or maybe generalized. Systemic symptoms such as fever, fatigue and weight loss may be present [2].

Ascites was never reported. Only three cases presenting with facial edema have been reported from published literature searched in the last 10 years. They included a 30-year old female presenting with tremendous swelling of the face leading to misdiagnosis of contact dermatitis [6].

A two-year old male presenting with fever, pallor, alopecia and facial swelling most evident in the peri-orbital area [7].

A 19-year old female presenting with a three-year history of diffuse facial swelling misdiagnosed as a benign cutaneous condition by various practioners [8].

Although the pathogenesis of facial swelling is unclear, Lee et al, (2016 p.1-6) suggested in his report of eight cases of cutaneous lymphomas that the angiocentric and pannicultis-like pattern was associated with dermal edema manifesting as facial swelling. They suggested that a suspicion of cutaneous lymphoma should be entertained in cases of recurrent and refractory facial swelling [9].

Pleural effusion on imaging was reported in five of 83 patients reported by Willemze, 2008 [2]. In a review of clinical features of 21 cases of SPTCL, leukopenia, anemia and thrombocytopenia occurred in 52, 43 and 10 percent of patients, respectively. Two or more cytopenias were present in $29 \%$ and elevated lactate dehydrogenase in $73 \%$, factors which were correlated with fatal outcome [3].

The over-all five-year survival rate for SPTCL exceeds $80 \%$, however, concomittant hemophagocytic syndrome (HPS) found in $24 \%$ of cases reduces it to less than $50 \%$, specifically those with SPTL-AB. [2]. From the systematic analysis by Go in 2004, 36 (33\%) of 109 patients of SPTCL had HPS at the time of diagnosis [4]. Eighteen of them received anthracycline, eight of whom went into remission [4].

Though the treatment of malignancy-associated HPS should aim to control the overactive immune system and treat malignancy, Wang et al explained that there are no universal conclusions on whether an HLH-directed, malignancy-directed or combined approach should be adopted due to lack of prospective, randomized or controlled clinical trials [10].

As there is no standard treatment for subcutaneous panniculitic T-cell lymphomas, most regimens used are anthracycline-based with the combination of cyclophosphamide, doxorubicin, vincristine, and prednisone (CHOP) or CHOP-like combinations [11].

However, poor response to such regimen may require the use of other treatment protocols, the recommendations of which are very widely variable.SPTCL, rare as it is, may present with unusual clinical manifestations. HPS may complicate the course, treatment decisions and outcome of the disease.

\section{References}

1. Pizzo Philip A (2016) Principles and Practice of Pediatric Oncology, 7th Edition, Wolters Kluwer, Pediatric lymphoma
(1166).

2. Willemze R, Jansen PM, Cerroni L, Berti E, Santucci M, et al. (2008) Subcutaneous panniculitis like T-cell Lymphoma: definition, classification and prognostic factors: an EORTC Cutaneous Lymphoma Group Study of 83 cases. Blood Journal 111: 838-845.

3. Ghobrial IM, Weenig RH, Pittlekow MR, Qu G, Kurtin PJ, et al. (2005) Clinical Outcome of Patients with Subcutaneous Panniculitis - like T- cell lymphoma. Leukemia \& Lymphoma 46: 703-708.

4. Go RS, Wester SM (2004) Immunophenotypic and molecular features, clinical outcomes, treatments, and prognostic factors associated with subcutaneous panniculitis-like T-cell lymphoma: a systematic analysis of 156 patients reported in the literature. American Cancer Society 101: 1404-1413.

5. Chibuzo O'Suoji, Jennifer JG Welch, Sherrie L Perkins, Lynette M Smith, Sheila Weitzman, et al. (2016) Rare Pediatric NonHodgkin Lymphomas: A Report From Children's Oncology Group Study ANHL 04B1. Pediatric Blood Cancer 63:794-800.

6. Kosari F, Akbarzadeh Hosseini S (2014) Local Facial Edema: a Novel Presentation of Subcutaneous Panniculitis - like T-Cell Lymphoma in a 30-Year-Old Iranian Woman. Acta Medica Iranica 52: 950-953.

7. Chinello Matteo, Naviglio Samuele, Remotti Daniele, Locasciulli Anna, Ventura Alessandro (2015) Subcutaneous Panniculitis-like T-cell Lymphoma Presenting with Diffuse Cutaneous Edema in a Two-year-old Child, Journal of Pediatric Hematology Oncology 37: 329-330.

8. Mohan Varadanayakanahalli Bhojaraja, Pradeep Kumar Reddy Kistampally, Karthik S Udupa, Joseph Thomas (2016) Subcutaneous Panniculitis - Like T-Cell Lymphoma: A Rare Tumour. Journal of Clinical and Diagnostic Research 10: 29-30.

9. Lee WJ, Moon IJ, Won CH, Chang SE, Choi JH, et al (2016) Facial Swelling: An Atypical Presentation of Cutaneous Lymphoma. International Society of Dermatology 55: e440-e446.

10. Wang H, Xiong L, Tang W, Zhou Y, Li F (2017) A Systematic Review of Malignancy- Associated Hemophagocytic Lymphohistiocytosis That Needs More Attentions. Oncotarget 8: 59,977-59, 985.

11. Thakur A, Chaudhari S, Thakur M, Chaudhari P (2015) Subcutaneous Panniculitis-like T-cell Lymphoma: Review of Therapies. Internal Medicine: Open Access 5: 1-4.

Copyright: (02020 Paula Bianca E. Nuqui. This is an open-access article distributed under the terms of the Creative Commons Attribution License, which permits unrestricted use, distribution, and reproduction in any medium, provided the original author and source are credited. 